\title{
JEUX DE MOTS, CAUSES DE LÉGENDES
}

\section{Louis Deroy}

Liège

I. Parmi les multiples causes des mythes qui se sont développés chez les peuples anciens et qui se produisent encore dans les sociétés queilque peu primitives, le jeu de mots ne reçoit pas toujours la place qui lui revient. II ne s'agit pas, bien entendu, du jeu de mots spirituel, du calembour pour rire. II faudrait parler plutôt d'étymologie, en entendant par là non pas l'étymologie scientifique telle que l'établissent les linguistes modernes, mais celle que l'on qualifie souvent d'étymologie populaire' et qui est souvent une étymologie pseudo-savante pratiquée, sur des ressemblances apparentes de mots, par des poètes, des sages, des prêtres. En réalité, il faut se reporter à des mentalités prélogiques, précritiques, préscientifiques, dans lesquelles les hommes croient que les noms possèdent une puissance magique, une intention mystérieuse ou simplement une signification pseudo-historique plongeant dans un passé incontrôlable.

II n' est pas toujours nécessaire de remonter à une époque très lointaine, ni de s'adresser à des peuples peu civilisés pour prendre contact avec le phénomène. N'en cherchons d'autre preuve que le culte chrétien de saint Expédit, dont le succés domine encore actuellement une critique évidente. En vérité, nous ne savons pratiquement rien de ce saint, si ce n'est qu'il fut martyrisé en Arménie, peut-être au temps de Dioclétier. Mais les chrétiens du Moyen Age se sont avisés que son nom latin Expeditus signifiait 'dégagé, dispos, pront et, en conséquence, ils se sont mis à l'invoquer comme le patron des causes urgentes. IIs lui prêtèrent comme maxime le mot latin hodie 'aujourd'hui'. Des images pieuses le représentèrent foulant aux pieds un corbeau, symbole de la remise à plus tard, parce que, disait-on au Moyen Age, cet oiseau ne cesse de crier, en latin, cras cras, c'est-à-dire 'demain demain'1. Le nom du saint a suffi à créer son personnage

${ }^{1}$ Cf. Ch. Nyrop, Linguitique et histoire des moeurs, trad. par E. Philipot, Paris 1934, Ch. Nyrop, Lingti $155-157$.
. 
Dans la vallée belge de la Meuse, la légende de saint Materne, populaire encore au siècle dernier, a été enrichie depuis le Moyen Age par l'insertion de démêlés imaginaires avec un prétendu dieu Nam, dont on avait cru reconnaître le nom dans les toponymes Namur, Namèche et même Dinant ${ }^{2}$.

On sait que le nom flamand d'Anvers, Antwerp, encore employé au $16^{\text {e }}$ siècle comme nom commun signifiant 'digue', chaussée', a été populairement interprété comme un composé de hand 'main' et werpen 'jeter': cette étymologie a donné naissance à la légende de Brabo, qu'une statue monumentale sur la principale place publique d'Anvers illustre encore aujourd'hui ${ }^{3}$.

En Suisse, dans le voisinage de Zermatt, le nom de la localité d'Aroleit, qui procède d*Arolletum 'bois d'arolles', a' été interprété par les gens de langue alémanique comme un composé de aar 'aigle' et leid 'souffrance', et il en est sorti la légende de la mère à laquelle un aigle ravit son nourrisson couché sur le pré, légende mise en poésie par Gottfried Keller. A ces exemples encore proches de nous, on pourrait ajouter bon nombre d'autres, décelés dans diverses régions du monde et à différentes époques ${ }^{5}$. Ils montreraient bien l'universalité du phénomène.

2. C'est spécialement à la Grèce antique que la présente étude est consacrée. Comme le montre excellemment le Cratyle de Platon, les anciens Crecs, même les plus cultivés et peut-être surtout ceux-là, ont éprouvé une propension manifeste pour le jeu de mots étymologique. C'est un tour d'esprit invétéré, puisque déjà dans les poèmes homériques, les philologues en ont reconnu

- F. Rousseau, Fausses étymologies, créatrices de légendes, dans Mélanges Jean Haust, Liège 1939, pp. 357-375.

3 Légende rappelée par Ch. Nyrop, op. cit., pp. 165-166.

$4 \mathrm{~W}$. von Wartburg. Problèmes et méthodes de la linguistique, trad. par P. Maillard, Paris 1946, p. 113.

5 Voir notamment: A. F. Pott, Etymologische Legenden bei den Alten, dans "Philologus", Suppl. 2 (1861), pp. 251-348; O. Keller, Lateinische Volksetymologie und Verwandtes, Leipzig 1891, p. 217 et ss. (Volksetymologische Mythenbildung); A. Bertholet. Wortanklang und Volksetymologie in ihrer Wirkung auf religiösen Glauben und Brauch, dans "Abhandl. der Preuss. Akad. der Wiss.", phil-hist. KI., 1940, nr. 6; et les études déjà citées de Nyrop, Rousseau et von Wartburg. 
de multiples illustrations ${ }^{6}$. Il n'est guère douteux qu'un très grand nombre d'épisodes et de traits surprenants de la mythologie' grecque sont issus, en dernière analyse, de tels rapprochements "étymologiques" apparents, soit entre des mots grecs, soit - et la démonstration est évidemment plus difficile à faire entré des mots préhelléniques. Ainsi, comme on le sait depuis longtemps, c'est de jeux de mots qu'on est parti pour imaginer qu'Aphrodite ('Apeodítm) était née de l'écume de la mer

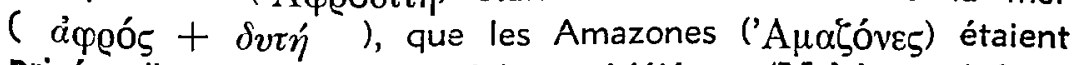

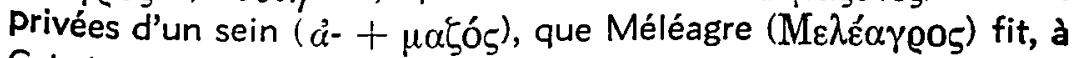
Calydon, une chasse de malheur ( $\mu \varepsilon \lambda \hat{\varepsilon} \alpha+\ddot{\alpha} \gamma \varrho \alpha$ ), et ainsi de suite. Mais si ces exemples sont bien connus en général, il en reste assurément encore un grand nombre d'autres à découvrir et ce sont naturellement les moins apparent: Or, il importe de déceler ces éléments adventices et secondaires des mythes, si l'on veut éviter de partir à faux dans une exégèse plus profonde. Pour illustrer ce principe, je me propose, dans la suite de cet article, de signaler quelques cas encore méconnus, où la linguistique permet de réaliser, au préalable, un certain 'déshabillage' mythologique.

3. La légende grecque voulait que le grand Héraclès fut mort empoisonné pour avoir revêtu une tunique de cérémonie que sa femme Déjanire lui avait remise. Pour expliquer cet accident, les mythographes ajoutent que Déjanire avait préalablement imprégné la tunique d'un violent poison, croyant qu'il s'agissait d'un philtre d'amour, comme le lui avait dit trompeusement le centaure Nessus. Je pense que cette justification a été introduite après coup. II est tout à fait possible, en effet, qu'à l'origine du mythe, on ait joué simplement sur l'homonymie de deux mots *áoj $\alpha_{00_{5}}$, l'un désignant un très fin tissu de lin employé pour confectionner des vêtements de dessous recherchés, l'autre étant le nom d'une redoutable plante vénéneuse à la saveur brulante, problablement l'hellébore blanche. Ainsi, à l'insu de l'innocente

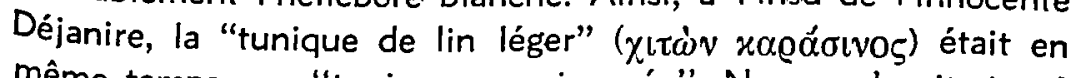
même temps une "tunique empoisonnée". Nessus n'avait rien à

6 Cf. L. Ph. Rank, Etymologiseering en verwante varschijnselen bij Homerus, Assen 1951, 158 Rp. 
faire primitivement dans cet épisode, à moins justement que le méchant conseil qu'il donna à Déjanire pour se venger, n'ait été, sous sa forme première, de donner à Héraclès un $\chi \iota \tau \dot{\omega} v$ zaojáalvoร. On comprend mieux ainsi que Déjanire ne se soit pas méfiée. Dans la version classique, l'auditeur avait le droit de se demander comment Nessus, mourant en sa fonction de passeur, avait eu le temps de confectionner et de remettre à Déjanire, sous le regard courroucé d'Héraclès, cet étrange philtre composé de sperme et sang, et comment Déjanire avait pu le conserver et s'en servir aussi naïvement. Le jeu de mots, au contraire, qu'il ait été grec ou déjà préhelléniquē, était réputé irrémédiablement trompeur. Nous retrouvons, dans la version du mythe que je crois la plus ancienne, la malice qui caractérise aussi les meilleures réponses des oracles grecs.

4. Quand, dans la légende des Argonautes, Jason et ses compagnons se donnent ou se voient imposer comme mission de conquérir la "toison d'or", on a le droit, sans fausse naïveté, de trouver surprenante et même saugrenue l'idée d'introduire cette toison au centre même du récit. II s'agissait, expliquent les mythographes, de la toison du bélier qui servit de monture à Phrixos lors de son arrivée en Colchide. C'est une jolie façon de rattacher la légende de Jason à celle de Phrixos. Mais, à mon avis, le raccord est artificiel et secondaire. II ne suffit pas à justifier le choix de la toison comme objectif suprême du voyage de Jason.

Me ralliant partiellement à des théses déjà formulées, je pense que dans la légende poétisée des Argonautes survit le lointain souvenir des expéditions maritimes menées, dès le début du deuxième millénaire avant notre ère, par les marins égéens, notamment par ceux de la Crète minoenne, pour aller chercher en Colchide, c'est-à-dire au pays des Hourrites caucasiens, les lingots de bronze que cette région était alors seule à produire grâce à la richesse de ses mines et à l'art de ses fondeurs. Mais le caractère commercial et utilitaire de ces navigations n'était guère compatible avec la poésie et l'imagination fertile des con-

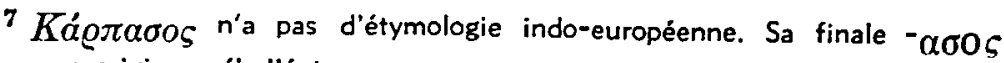
évoque une origine préhellénique. 
teurs de légende: II va nous suffire de déceler un jeu de mots, involontaire cette fois, pour découvrir comment on a glissé du réel dans lé merveilleux, avec la même propension qui a poussé des conteurs français presque modernes à prêter à Cendrillon des chaussures de verre au lieu de ises pantoufles de vair.

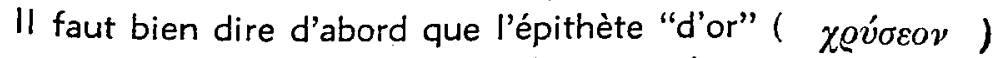
de la toison colchidienne est secondaire et n'a pas, pour nous, d'importance. Dans la vieille poésie grecque, toutes sortes d'objets et même d'animaux, qui avaient un caractère divin ou merveilleux, sont ainsi réputés "d'or", sans qu'il faille songer à la présence effective de ce métal. Hérodote d'ailleurs néglige l'épithète quand, dans un passage de ses Enquêtes (VII, 193), il fait allusion au

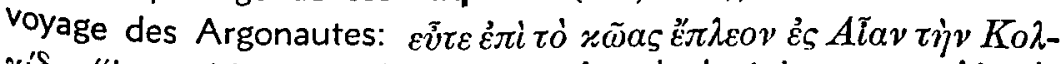
$\chi_{i}^{\prime} \delta \alpha$ "Iorsqu'ils naviguèrent en quête de la toison vers Aia de Colchide". Quant à la toison elle-même, je pense que le nom qui la désigne traditionnellement, $w \tilde{\omega} \alpha \zeta$, nous fournit la clef du problème qui nous occupe. A côté de xic̃as, en effet, on trouve, en grec, quelques termes fort semblables de forme et cependant tout différents de sens: $\% \bar{\omega} 0_{5}$, puis par contraction $\psi \bar{\omega} \zeta$ "trou, creux, cavité, terrier, caverne" (c'était le nom propre d'une vieille prison souterraine à Corinthę) 8 , et $\tau \dot{\alpha} x \tilde{\omega} \alpha$ "les faces creuses ou concaves de l'osselet"'. Ce sont, dans l'usage classique, des termes plutôt archaïques et l'on peut croire qu'ils ont été plus répandus à l'époque préclassique, comme le suggère notamment l'emploi dans la toponymie corinthienne. Cela étant dit, on imagine Sans peine que, dans sa version originelle, la légende des Argonautes employait une expression signifiant "en quête des mines" ( $\dot{\varepsilon} \pi i \tau \dot{\alpha} x \omega \tilde{\alpha} \alpha$ ou quelque chose de semblable, soit en grec, soit en préhellénique), mais qu'ultérieurement les conteurs de légendes, suivant peut-être en cela leur auditoire mal renseigné sur la métallurgie colchidienne primitive, se mirent à comprendre "en quête de la toison" "̇̉i $\tau \dot{\delta} x \tilde{\omega} \alpha \varsigma$ ) ou même d'abord "en quête

8 Hésychius atteste une graphie xóos.

9 Ce sont vraisemblablement des survivances du préhellénique. Le latin a conservé, sans doute par l'intermédiaire de l'étrusque, des formes correspondantes: d'une part, coum (cohum) "trou, cavité, voute céleste", qui représente un ancien "couom, et l'autre part, cavus "creux, creusé", caverna "souterrain, Caverne, terrier, tanière". 
des toisons" (દ'ji $\tau \dot{\alpha} \varkappa \omega ́ \varepsilon \alpha)$. Le glissement sur un mot ouvrait la porte à l'inattendu et au merveilleux: c'était trop beau pour que le; poètes n'en fissent pas leur profit.

5. Comme il est normal, confusions et jeux de mots se sont surtout produits quand les mots étaient isolés, quand ils ne faisaient pas clairement partie de groupes lexicaux familiers. C'ejt pourquoi les emprunts passés isolément du préhellénique au grec ont souvent fourni l'occasion d'étymologies populaires et, par là, de mythes ${ }^{10}$. J'en vois une belle illustration dans la légende des Cyclopes.

Il existait, dans la langue préhellénique, un mot "kala "pierre". Nous avons le droit de le supposer parce qu'il en subsiste, grec ancien, diverses attestations telles que $\chi \alpha \alpha^{\prime} \lambda \xi \xi$ "petite pierre, caillou, gravier" et $\chi \lambda .0 .00 ́ v, x \lambda \hat{\alpha} 0_{5}$ et $x \lambda \tilde{\eta} \varrho 0_{5}$ "caillou utilisé pour tirer au sort, lot'; aussi parce que cette 'base' *kala, répandue dans la Méditerranée occidentale par la colonisation égéenne, a survécu dar.s les mots latins calculus 'caillou' et glarea gravier'11, italiens calestro ou galestro 'terre pierreuse', français chail "pierre", chaillot "terrain pierreux", caillou, galet, clapier", irlandais gall "pierre, rocher" galgal "sorte de tumulus" (mot passé dans la terminologie archéologique), ainsi que dans beaucoup de toponymes ${ }^{13}$.

Or, le grec a conservé au.si le souvenir d'une forme préhellénique redoublée "kakala signifiant "grosse pierre": cette forme

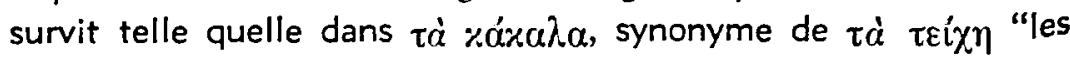
murailles, les remparts" 14 , et munie d'un suffixe diminutif dans $x_{\alpha}^{\alpha} \chi \lambda \eta \xi \xi$ "moellon, gros caillou", puis simplement "caillou, galet,

10 Sur le principe, voir mon ouvrage L'emprunt linguistique, Paris 1956. pp. 280-288.

11 L'emprunt de ces mots au grec est une hypothèse possible, mais qui se heurte à bien des difficultés.

12 Les survivances romanes sont beaucoup plus nombreuses. Je ne cite ici que quelques exemples bien connus.

13 On trouve des attestations de cette "base" préindo-européenne jusque dans les langues dravidiennes de l'Inde. L'étude de "kala comporte aujourd'hui une copieuse bibliographie. On y rattache souvent "kala au sens d" endroit abrité". Un exposé plus détaillé de cette question se trouve dans mon ouvrage Aspects linguistiques de la protohistoire européenne (à paraltre bientôt).

14 Eschyle, fragment 166 Nauck (cité par Hésychius). 
gravier"15. Le nom des Cyclopes, $K v^{\prime} \approx \lambda \omega \pi \dot{\varepsilon}$ s, procède, à mon avis, d'un dérivé préhellénique de *kakala. Dans la tradition grecque, en effet, les Cyclopes apparaissent comme des hommes primitifs, non civilisés ${ }^{16}$, d'une grande force physique, capables, comme Polyphème dans l'Odyssée $(X, 243,481,537)$, de mouvoir et même de lancer très loin d'énormes blocs de rocher. En particulier, ils sont tenus pour les constructeurs des murailles en gros blocs juxtaposés que l'on voyait en divers endroits de la Grèce, notamment à Mycènes et à Tirynthe, murailles que I'on qualifiait, justement

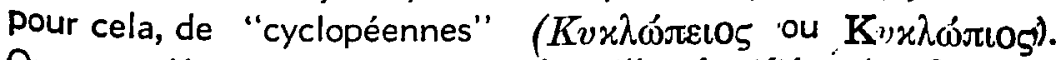
Or, pour désigner les enceintes des villes fortifiées, les Grecs ne disaient pais seulement $\tau \varepsilon i ́ x \eta$ et $x \alpha ́ x \alpha \lambda \alpha$, mais ils se servaient aussi de deux mots dont le sens propre est "cercle, rond",

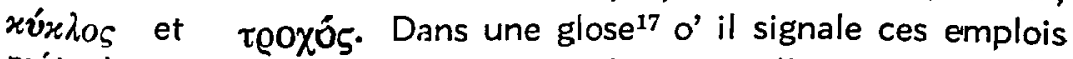
métaphoriạues, le lexicographe Hésychius rappelle une expression qui se trouvait dans l'Héraclès, pièce aujourd'hui perdue, de Sophocle ${ }^{18}$, et qui est suggestive pour notre propos: Kvincítios

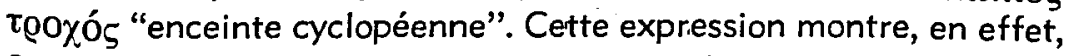
ccmment, à une époque archaïque et sans doute prélittéraire, une etymologie populaire a rapprcché $x \alpha \dot{\alpha} x \alpha \lambda \alpha$ de $x \dot{x} x \lambda \alpha_{\text {s, }}$ et a

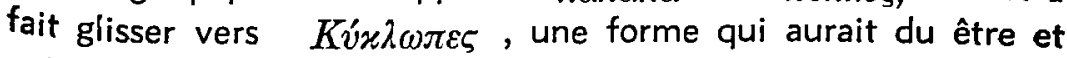

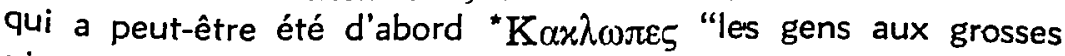
pierres". A partir de là, l'étymologie populaire s'est développée.

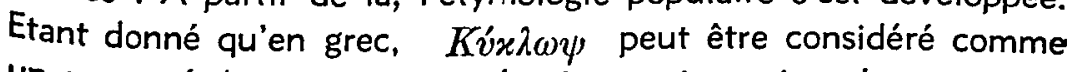
un composé de $x u ́ x \lambda o s$ et de $\omega^{\prime} \pi$, qui exprime la notion de "voir"19, on en vint finalement à imaginer les Cyclopes non pas cela aurait été peu original -- comme de.s "êtres aux yeux ronds", mais comme de véritables monstres munis d'un oeil circulaire au milieu du front. Cette conception était déjà si familière avant

15 Ce mot présente aussi une variante $\chi 0 ́ \chi \lambda \alpha \xi$, visiblement altérée par un rapprochement populaire avec $x o ́ \chi \lambda{ }_{0}$ et $x 0 \chi \lambda \hat{i}_{\alpha 5}$ "coquillage, coquille". Cf. la remarque de paul-Festus, 35,4 , à propos de coclaca.

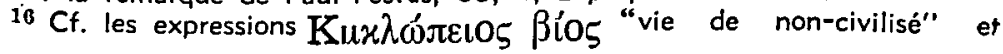
$K v \times \lambda \omega \pi \iota x \tilde{\omega} s \quad \zeta \tilde{\eta} v$ "vivre à la sauvage".

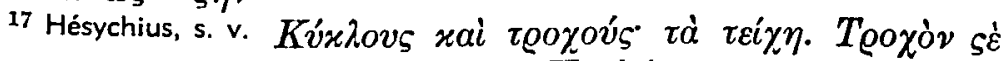

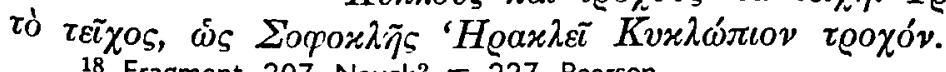

1918 Fragment $207 \mathrm{Nauck}^{2}=227$ Pearson.

19 Cf. P. Chantraine, La formation des noms en grec ancien, Paris, 1933, p. 256 , qui cite aussi $\varepsilon \lambda i x \omega \psi, \operatorname{s} \iota \nu \omega \pi o ́ s, \beta o \tilde{\omega} \pi \iota \varsigma, \gamma \lambda a v \tilde{\omega} \pi s, \varepsilon v \omega \pi o ́ s$,
etc. 
Homère que celui-ci a pu, sans préalable, broder sur le thème dans l'épisode fameux de l'Odyssée: l'aveuglement de Polyphème.

6. Le mot préhellénique "kala "pierre" se trouve aussi, et cette fois dès l'époque minoenne, à l'origine d'un trait curieux du mythe de Cronos. Hésiode déjà, dans sa Théogonie (vers 459 et ss.), puis d'autres mythographes grecs racontent qu'à l'origine du monde, Cronos, craignant d'être un jour détrôné par un de ses enfants, se débarrassait de ceux-ci dès leur naissance en les avalant. Lassée dé ce comportement (on le serait à moins), son épouse Rhéa s'arrangea pour s'accoucher en cachette. Puis, une fois le nouveau-né (c'était Zeus) mis en sécurité, sa complice Gaia plaça sur les genoux du père, selon le rite requis pour la reconnaissance, une grosse pierre entourée de langes, qui fut aussitôt avalée. Pourquoi donc le conteur a-t-il choisi, comme objet de substitution, quelque chose d'aussi différent d'un enfant que l'est une pierre? Et comment a-t-il pu faire admettre à ses auditeurs l'idée qu'un dieu, fut-il notoirement glouton, avait été trompé par un subterfuge aussi grossier? En réalité, compte tenu d'une mentalité encore primitive, l'erreur était rendue inévitable par l'intervention d'un autre élément: la puissance magique du mot. II faut se placer dans l'état d'esprit d'hommes qui ne distinguent pas le nom de la chose désignée, pour qui le nom prononcé ou écrit est censé produire réellement la présence de la chose. Dans le cas de Cronas, le fait linguistique, jusqu'ici insoupçonné, qui a justifié la légende, c'est qu'à côté de "kala "pierre", il existait, en préhellénique, un autre mot "kala ou "kale signifiant "enfant". $\mathrm{Ce}$ second mot, il est permis de le restituer à partir de plusieurs survivances, qui n'ont pas, que je sache, été bien expliquées autrement:

1) $x \alpha \bar{\lambda} v \xi \xi-\mu \gamma O \zeta$ "embryon, foetus, nouveau-né". Ce mot est

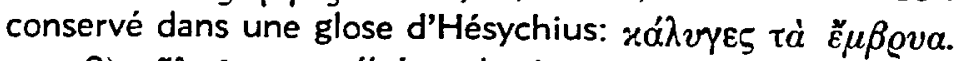

2) $x \alpha \tilde{\alpha} \lambda v \xi$-vxo丂 "plantule, jeune, pousse, rejeton, bouton"20.

20 Les articles des différents dictionnaires grecs rapportent traditionnellement plusieurs significations inexactes. Celles qui sont citées ici, sont celles qui me paraissent ressortir d'un examen direct des textes: "plantule encore en terre" (Soph., O. R., 25; Ar., Ois., 1065), "jeune pousse sortant de terre" (Eschyle, Ag., 1392; Hdt., 3, 100; Théophr., H. P., 8, 2, 4), "rejeton" (Hdt., 2, 92), "bouton de fleur", (H. hom. Aphr., 427; Cratinos, 98; Aristt., H. A., 554 a 12; Théophr., H. P., 4, 10, 3; Théocr., 3, 23; Anth., 5, 163; Hsch.,

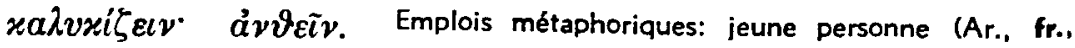

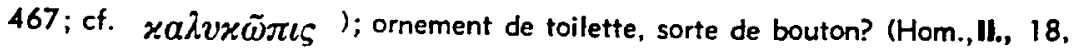
401 ; H. hom. Aphr., 87 et 163). 
Ce mot n'est qu'une variante du précédent. II comporte un suffixe issu du préhellénique ${ }^{*} \mathbf{k e / - k i}$, qui exprimait la relation et la similitude. Le prototype préhellénique aurait été *kale-ke "analogue à un embryon, à un nouveau-né, à un enfant".

3) $x \varepsilon ́ \lambda \omega \varrho$ "enfant", $x \varepsilon \lambda \omega ́ \varrho \iota 0 v$ "petit enfant". Rappelons que le changement de timbre vocalique $a>e$ a tendu à se produire, dans des conditions encore indéterminées, sur un vaste domaine méditerranéen comprenant aussi bien les parlers asianiques.que la langue égéenne (y compris l'étrusque) ${ }^{21}$.

4) veoyĩiós "nouveau-né". Ce terme, rare et poétique, est déjà attesté dans l'Odyssée XII,86) en parlant d'un chiot. L'i peut être un allongement métrique à l'origine.

5) Étrusque clan (pluriel, collectif ou indéfini clenar) "enfant, fils". Alfredo Trombetti22 a déjà suggéré de rapprocher clan de

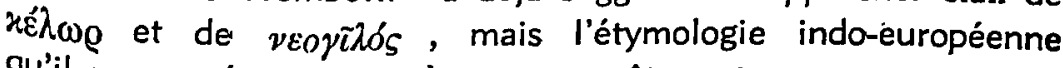
qu'il a proposée, ne peut, à mon sens, être retenue.

6) Probablement $-x \lambda \varepsilon \eta_{\zeta}>-x \lambda \tilde{\eta} S$ comme second élément d'anthroponymes. C'est clair dans le nom d'Hercule. A l'origine, il faut partir de "Hera-kale "enfant de Hèra", c'est-à-dire fils de la Grande Déesse Terre. L'effet de l'accent d'intensité initial tendait, en préhellénique, à fermer et même à amuir les voyelles des syllabes internes: c'est ce qui a finalement provoqué une prononciation *Herekele, représentée par l'étrusque Hercle (et *Hercule, si l'u du latin Hercules n'est pas une anaptyxe). Mais, du côté grec, la syncope semble avoir été partiellement empêchée parce que les locuteurs avaient gardé le sentiment que le premier terme était le nom de la déesse Hèra. Quant au second terme *-kle, les Grecs l'ont rapproché, par étymologie populaire, des mots

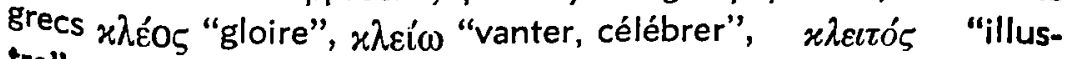

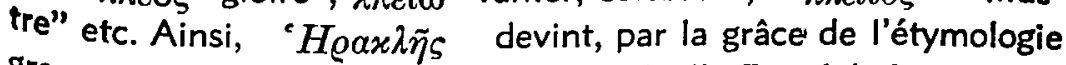
grecque, "celui qui fait la gloire d'Hèra". En réalité, on peut croire qu'Hèra était bien primitivement la mère d'Hercule. Lors

21 II faut en distinguer les homonymes $\chi \varepsilon ́ \lambda \omega \varrho$ "cri" et $\chi \varepsilon ́ \lambda \omega \varrho$ "castrat, eunuque", qui ont des origines tout à fait différentes. Cf. E. Boisacq, Grec หĚ $\omega_{Q}$ "fils" et homonymes, dans les "Mémoires de la Société de linguistique de Paris", 17 (1911), pp. 113-116.

22 La lingua etrusea, Florence, 1928, p. 6. 
de sa descente rituelle aux enfers, c'est du lait de son sein que le héros, déjà barbu, mais toujours dieu-enfant de la triade divine, était censé tirer la revivification nécessaire à son retour parmi les vivants ${ }^{23}$. Sur un miroir de Volterra du Ille siècle avant J.-C., qui représente cet allaitement symbolique ${ }^{24}$, l'inscription étrusque est significative: Hercle Unial clan"is "Hercule fils de Uni", c'està-dire de la déesse qui, en Etrurie, correspond à la romaine Junon et donc à la grecque Hèra ${ }^{26}$. Mais les Etrusques ne connaissaient plus Hèra sous ce nom et, par conséquent, le sens d'Hercle leur échappait: c'est ce qui explique l'adjonction d'Unial clan, qui nous fournit une indication d'autant plus précieuse qu'elle est une traduction involontaire. Le second terme préhellénique - ${ }^{*} \mathbf{k l e}$,

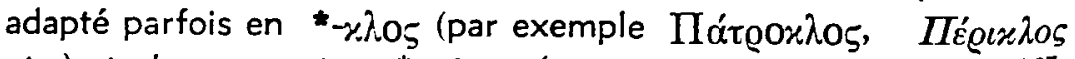

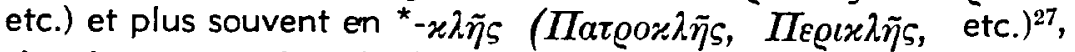
s'est largement répandu dans l'anthroponymie grecque. Sans tenir compte de la valeur surprenante qu'on lui prêtait à l'époque classi-

23 Cf. en dernier lieu $W$. Déonna, Deux études de symbolisme religieux, Bruxelles-Berchem 1955 (coll. "Latomus", XVIII), pp. 5-50: La légende de Pero et de Micon et l'allaitement symbolique. La signification symbolique de ce rite préheliénique se retrouve pareille dans l'ancienne Egypte: cf. J. Leclant, Le rôle du lait et l'allaitement d'après les textes des pyramides, dans "Journal of the Near Eastem Studies", 10 (1951), p. 123 et ss.

24 P. Ducati, L'arte etrusca, tav. 241, fig. 588; G. Giglioli, L'arte etrusca, tav. 299, fig. 5; tav. 300; "Studi Etruschi", 13 (1939), p. 252.

25 G. Buonamici, Epigrafia etrusca, Florence 1932, p. 390, tav. LIV; $M$. Buffa, Nuova raceolta di iserizioni etrusche, Florence 1935, n. 288; M. Pallottino. Testimonia linguae etruscae, Florence 1954, n. 299. Ct. Déonna, op. cit., p. 19 et 31 .

26 Le rapport de $-\chi \lambda \hat{\eta} \varsigma$, dans ' $H \varrho \alpha x \lambda \tilde{\eta} \varsigma$, avec l'étrusque clan a été aperçu par Maresch dans les "Mitteilungen des Vereins klassischer Philologen in Wien", 7 (1931), pp. 50-55 (d'aprés P. Kretschmer dans "Glotta", 21 (1933), p. 194). Mais, à cause du mythe, semble-t-il, Maresch n'a pas osé admettre le sens de "fils" et il suppose une signification dérivée "accepté comme un fils", qu'il voudrait aussi retrouver dans le latin cliens.

27 Cf. les listes données par H. Usener, Die Sintfluthsagen, Bonn 1899 , pp. 51-58. 
que, je crois pouvoir affirmer que, dans les noms les plus anciens, il avait la même signification et la même fonction que - $\pi 0 u \lambda_{0}$ en grec moderne, que -son, -sohn, -zoon etc. dans les langues germaniques ${ }^{28}$.

Ces rapprochements suffisent, je crois, à rendre vraisemblable l'existence, en préhellénique, d'un mot *kale ou *kala "enfant, jeune enfant", et, dès lors, on aperçoit, en se replaçant dans une mentalité suffisamment primitive, comment Cronos, abusé par l'homonymie, a pu prendre réellement une pierre pour un nouveau-né. Il a été victime de la puissance des mots. C'est à cette condition linguistique seulement que la substitution a été tenue pour possible et la légende pour acceptable ${ }^{29}$.

7. Cette exégèse apporte naturellement un nouvel appui à l'explication toute pareille du mythe de Deucalion et Pyrrha. On sait que Deucalion, le Noé grec, se retrouvant après le déluge en la seule compagnie de sa femme Pyrrha ${ }^{30}$, repeupla, dit-on, la terre par un moyen inattendu que Zeus lui avait indiqué: en jetant des pierres par-dessus son épaule. Toutes les pierres qu'il jeta, devinrent des hommes, et toutes celles que jeta Pyrrha, se changèrent en femmes. II est probable que cette métamor-

28 Sans me lancer dans un commentaire linguistique qui serait long et sans doute non décisif, je me borne à citer aussi deux autres mots qu'on pourrait songer à rattacher pareillement à la base *kala/“kale: ce sont gr. "petit enfant, bébé" (cf. il., 8, 164) et "pupille de l'oeil", et lyc. qla "lignée, race, famille" (ce dernier mot est rapproché de l'étr. clan par E. Vetter, Etruskische Wortdeutungen, Vienne 1937, P. 5 et ss.).

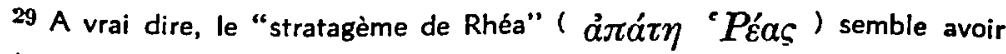
résisté à la sagacité des historiens des religions. Ch. Picard, dont on connait l'extraordinaire information, se borne à écrire dans son traité Les religions préhelléniques (Crète ef Mycènes) (Paris 1948), p. 117: "le mythe peut couvrir le résidu d'une lex sacra, comportant l'offrande rituelle du nouveau-né, et la pierre de substitution, jetée comme appât à la boulimie meurtrière du père, s'expliquerait alors par un fétichisme primitif: cf. les concrétions du Petit Palais Cnossien, en forme de déesse courotrophe, et la pierre dite de Cronos adorée à Delphes". A propos du même épisode mythique, il parle p. 118 de "thème folklorique crétois, assez primitif". ${ }^{30}$ Je suis tenté de supposer que, dans la version primitive, Deucalion
était tout à fait seul et dès lors dans l'impossibilité même de procréer normalement des enfants. On s'explique mieux, dans cette situation, le recours à Zeus et l'utilisation d'un moyen miraculeux. 
phose a été justifiée, à l'origine, par un jeu entre le nom de la

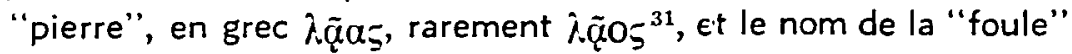
ou du "peuple", en grec yaós, comme les anciens Grecs mêmes l'ont cru, apparemment dès l'époque homérique ${ }^{32}$. A moins que quelque savant mythographe grec n'ait simplement rajeuni le

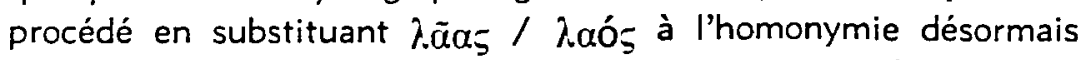
imperceptible d $\epsilon^{\prime}{ }^{*}$ kala / kale $^{33}$. Peu importent, après tout, les mots sur lesquels on a joué: une fois connu le procédé, il suffisait d'un peu d'astuce pour multiplier les exemples.

8. Il existe sans doute encore bon nombre d'autres cas, insoupçonnés ou du moins mal éclaircis, dans les multiples récits de la mythologie grecque. Je ne crois pas qu'ils soient tous faciles à déceler et à démontrer, mais il était, de toute façon, utile de souligner encore une fois l'importance du jeu de mots comme source de légendes, en particulier contre l'opinion de ceux qui considèrent a priori ce procédé comme négligeable ou même méprisable en

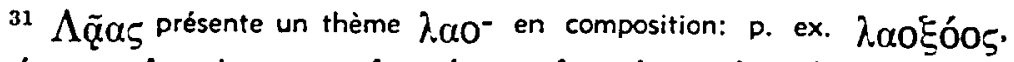

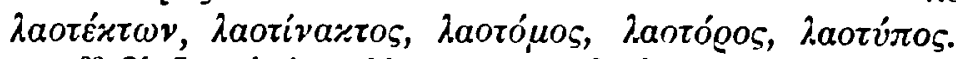

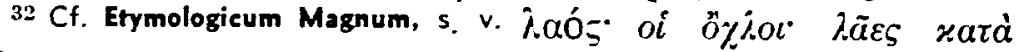

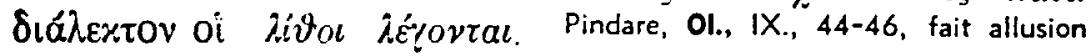

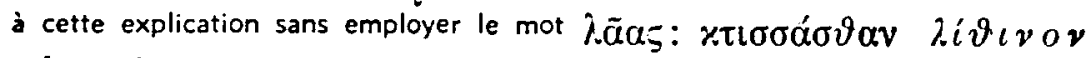

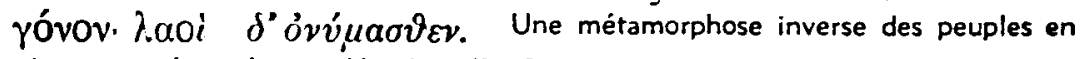
pierres est évoquée par Homère, II, 24,611

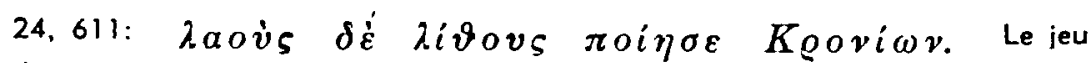
de mots était donc connu et utilisé dès alors. A vrai dire, il a pu être déja préhellénique. puisque les deux mots grecs pourraient bien procéder d'une même racine égéenne "law- (lap-) "couper, trancher". Cf. mon article La valeur du suffixe préhellénique - $n$ th- d'après quelques noms grees en -- $v \vartheta 05$, dans "Clotta", 35 (1956), p. 173 et 55.

33 L. Ph. Rank. op. cit., p. 103, pense que cette origine est impossible, parce que le mythe des hommes nés d'un jet de pierre se retrouve dans des légendes non-grecques (il renvoie à l'ouvrage de $H$. Usener, Sintfluthsagen, Bonn 1899, Pp. 245-256). Mais la question de l'expansion du mythe se pose tout différemment si, dépassant le monde grec, on fait remonter le jeu de mots à la langue préhellénique et, en même temps, aux parlers asianiques apparentés, et si l'on date la création du mythe d'avant le milieu du deuxième millénaire avant I. $-\mathrm{C}$. 
comparaison des prétendues grandes causes psychologiques, philosophiques ou même psychanalytiques.

En vérité, la plupart des mythes grecs sont des agrégats complexes qui se sont constitués progressivement et il est illusoire de prétendre les interpréter d'un seul coup et globalement. II faut d'abord les démonter, en éliminer les traits secondaires en recourant à divers indices, notamment à ceux qu'on peut tirer des mots. Négliger ce préalable, c'est risquer de mettre la charrue avant les boeufs, comme font des théoriciens de l'histoire de l'art qui mettent au compte de l'inspiration et de l'esthétique pure, telle attitude ou tel geste d'une statue, sans nous dire d'abord (et sans remarquer peut-être) que le sculpteur a du avant tout se soumettre aux nécessités techniques de la taille du marbre ou du moulage du bronze. 